\title{
Multimodality imaging findings of visceral myopathy in a child presenting with palpable abdominal mass
}

\author{
Zuhal Bayramoğluํㄹ, Ravza Yılmaz ${ }^{1}$, Ali Aslan Demir ${ }^{1}$, Çigdem Ataizi-Çelikel ${ }^{2}$, Faruk \\ Erdem Kombak ${ }^{2}$, Aygün İkinci ${ }^{2}$, Ensar Yekeler ${ }^{1}$ \\ ${ }^{1}$ Department of Radiology, Istanbul University Istanbul Faculty of Medicine; ${ }^{2}$ Department of Pathology, Marmara University \\ Faculty of Medicine, Istanbul, Turkey. E-mail: incezuhal@yahoo.com \\ Received: 15th August 2017, Revised: 5th January 2018, 21st January 2018, Accepted: 28th January 2018
}

SUMMARY: Bayramoğlu Z, Yılmaz R, Demir AA, Ataizi-Çelikel Ç, Kombak FE, İkinci A, Yekeler E. Multimodality imaging findings of visceral myopathy in a child presenting with palpable abdominal mass. Turk J Pediatr 2019; 61: 120-125.

Visceral myopathy is a rare cause of intestinal obstruction characterized by intestinal dysmotility and constipation. Patients often present with recurrent abdominal pain, vomiting and abdominal distension. We report a rare case of visceral myopathy in a child presenting with intraabdominal mass. We aimed to describe ultrasound, computed tomography and magnetic resonance enterography findings of this rare disease that has not been demonstrated before. Differential diagnosis of mural thickening with distinguishable layers in addition to intestinal dilatation in the absence of mesenteric inflammation includes visceral myopathy.

Key words: visceral myopathy, intestinal obstruction, ultrasound, magnetic resonance imaging, computed tomography.

Intestinal pseudo-obstruction (IPO) is a heterogeneous clinical syndrome caused by either intestinal or non-intestinal disorders 1. The primary form of IPO may be caused by defects of myenteric plexus nerves and ganglions (visceral neuropathy) or smoothmuscle degeneration and fibrosis (visceral myopathy, $(\mathrm{VM}))^{2}$. VM can be idiopathic (primary), familial transmitted via a dominant or recessive gene or associated with other diseases such as scleroderma, multiple sclerosis, systemic lupus, amyloidosis, Ehlers-Danlos syndrome, stroke and encephalitis. ${ }^{3}$ Clinical findings in patients with VM are nonspecific. Patients may be totally asymptomatic, but may also present with nausea, vomiting, postprandial pain, abdominal distension, diarrhoea and intestinal obstruction attacks which are seen in very few patients. ${ }^{4}$ As far as we know, abdominal mass as an initial presentation of visceral myopathy in a child is unexpected and has not been reported. We aimed to demonstrate this confusing entity with multimodality imaging findings that have not yet been described in the literature.

\section{Case Report}

A palpable abdominal mass localized in the left upper quadrant was examined in a three year-old male patient who was referred to the radiology department. There was no history of previous disease. He was in the $25^{\text {th }}$ percentile for height and weight. Remaining physical exam was found to be normal. Complete blood count, levels of serum electrolytes and albumin, and acute phase reactants such as $\mathrm{C}$ reactive protein and sedimentation, were found to be normal.

Abdominal ultrasound (US) examination revealed symmetrical concentric wall thickening of proximal jejunal loops throughout a long segment with detectable intestinal layers (Fig.1a). A smooth and normalised vascular network was found on the submucosal layer with Doppler ultrasound (DUS) examination suggesting developmental or degenerative processes rather than neoplastic entities (Fig. 1b). Hyperechogenic nodule formations located in the muscularis propria layer were depicted (Fig. 1c). Multiple reactive mesenteric lymph nodes, ovoid in shape, with the maximum short axis dimension of $8 \mathrm{~mm}$ were additional 


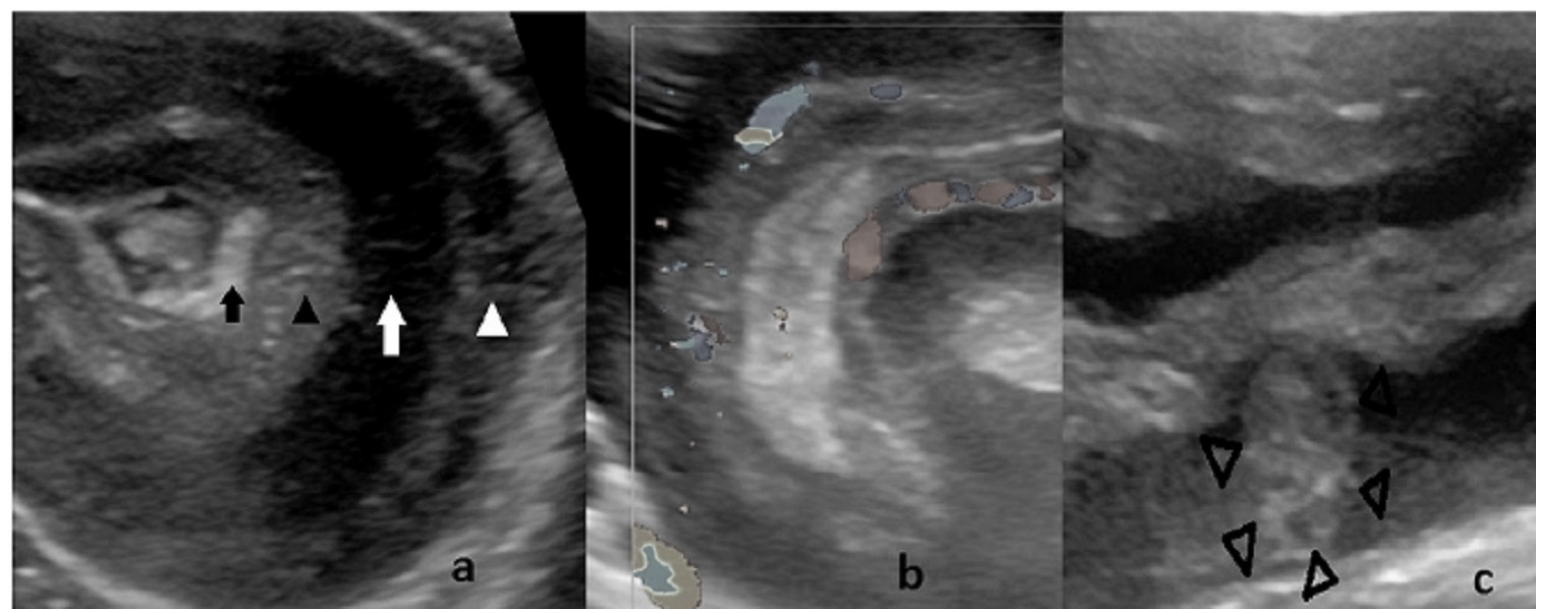

Fig. 1. a. Gray Scale Ultrasound Image: Sonographic evaluation reveals diffuse thickening of the involved jejunal segments with clearly visible mucosa (black arrow), submucosa (black arrow head), muscularis propria (white arrow), serosa (white arrow head) excluding infiltrative and neoplastic involvement. b. Doppler ultrasound image: Hypervascularity on submucosal layer due to enlarged vasa recta and increased blood flow to involved segments. c. Gray Scale Ultrasound Image: A hyperechoic nodule formation located in the muscularis propria layer was depicted suggesting a hyperplastic ganglia or a lymphatic aggregation (black arrow head).

US findings. Intravenous contrast enhanced abdominal computed tomography (CT) was performed in order to exclude extranodal lymphoma. Whole layers of involved jejunal segments were demarcated and significant submucosa and muscularis propria enlargement were found on CT (Fig. 2). Aneurysmatic dilatation or hyperdense mass formation as expected from intestinal lymphoma was not found. Mesenteric fat planes were free of inflammation but lymphangiectasia and associated mesenteric oedema was detected. We performed MR enterography (MRE) by

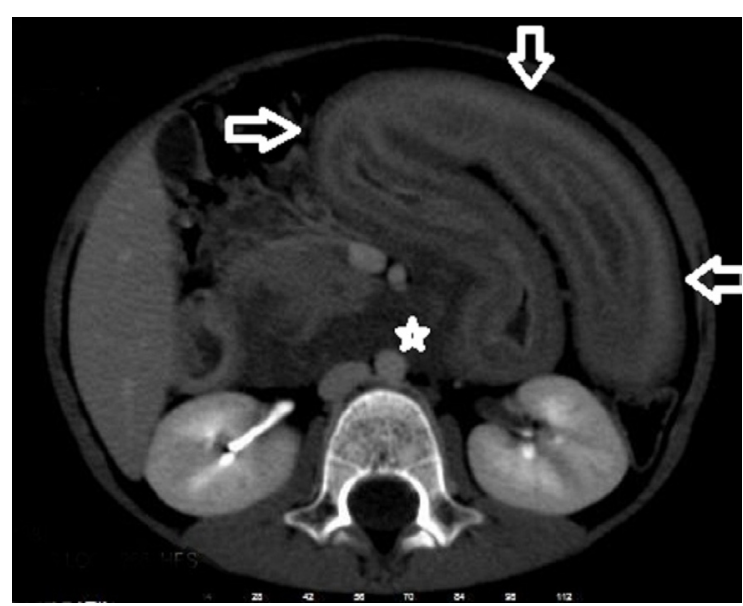

Fig. 2. Intravenous contrast enhanced axial section abdominal CT: Diffusely involved jejunal segments with demarkable mural layers were shown by closed arrows and additional mesenteric edema (asterix). using T2W- TRUFI (True FISP) sequence in order to demonstrate intestinal morphology and associated imaging findings. The duodenum was moderately dilated and proximal jejunal loops were diffusely thickened on MRE (Fig. 3a). Ileum and colonic segments presented normal signal and morphology. Mesentery of the thickened segments was hyper-intense on T2W- TRUFI sequence due to diffuse

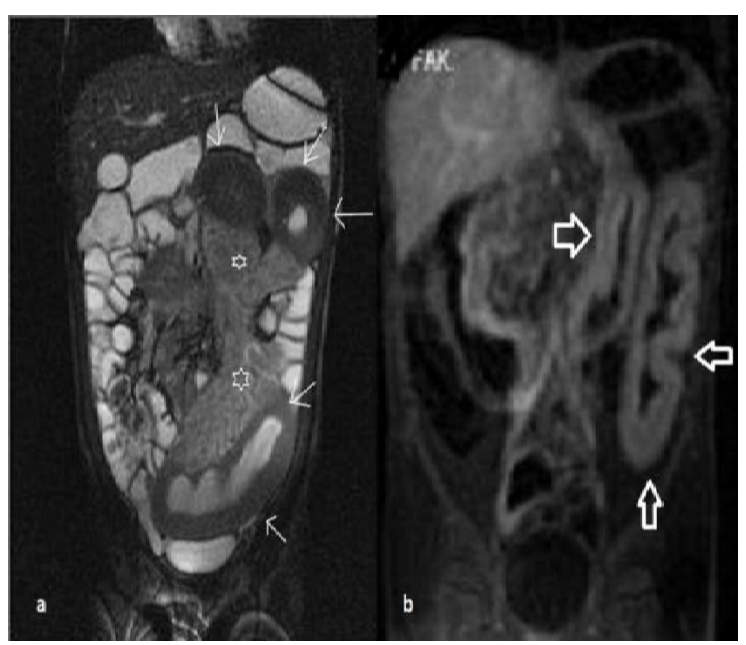

Fig. 3a. Coronal section of MR enterography (MRE) by T2W-TRUFI (True FISP) sequence. Jejunal segments were diffusely thickened on abdominal MRE (open arrow). Mesentery was hyper-intense due to lympangiectasia (asterix). b. Contrast enhanced $\mathrm{T} 1$ weighted fat saturated image shows intense mural enhancement without mesenteric inflammation. 


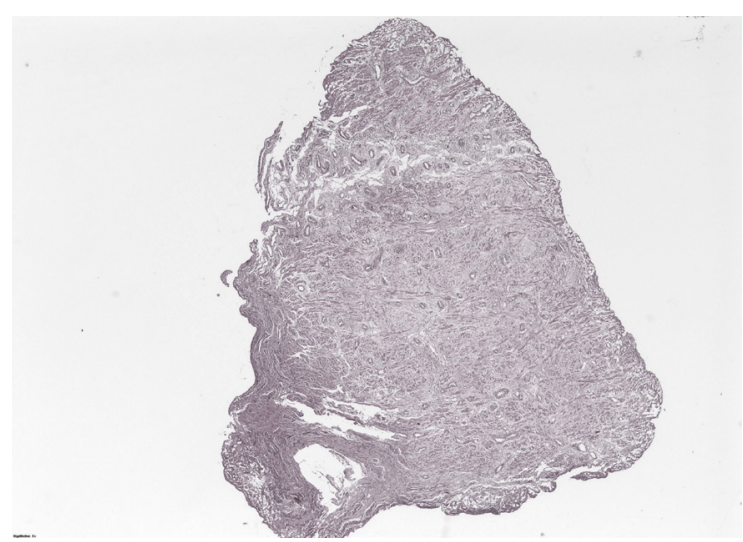

Fig. 4. Low power magnification of incisional jejunal biopsy, exhibiting thickened bowel wall (H\&E; x20)

oedema and accompanying lymphangiectasia (Fig. 3a). Contrast enhanced images on MRE showed diffuse intense contrast enhancement within the thickened loops, contrary to noninvolved intestinal segments (Fig. 3b). Since the intestinal layers were not obliterated and no mesenteric inflammation was found, radiological differential diagnosis included intestinal lymphangiectasia. The patient underwent colonoscopy two weeks after the radiological evaluation and histopathology revealed congestion and lymphoid aggregations in the large bowels. In order to achieve accurate diagnosis, the patient underwent surgical exploration three weeks after magnetic resonance imaging. Incisional jejunal biopsy regarding submucosa, muscularis and serosa (Fig. 4) exhibited neuronal hyperplasia, lack of ganglion cells and moderate interstitial fibrosis (Fig. 5). Dissection of muscle fibres by hyperplastic nerve bundles was striking (Fig $6 a, b)$. Immunohistochemistry was performed to highlight neuronal and muscular structures (Fig 7a, b). These morphological findings were found to be reminiscent of VM and clinical correlation was suggested. There was no clinical observation of neurofibromatosis as a differential diagnosis based on immunohistochemical staining. The patient has been under followup with symptomatic treatment regarding dietary rearrangement of five to six small meals a day, preferably a low-fat, low-fibre diet, and additional nutritional supplements due to hypovitaminosis, antibiotherapy in case of enteritis and intravenous hydration and nasogastric decompression if required. Followup ultrasound examinations within the year

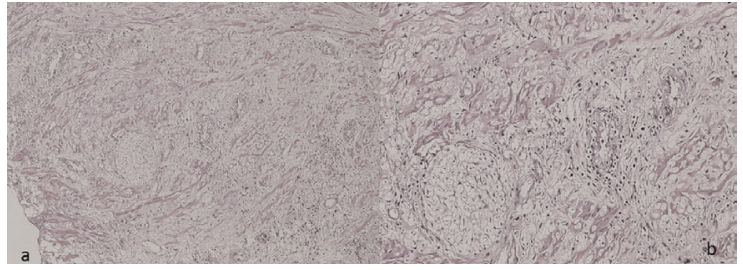

Fig. 5. Masson trichrome staining highlights mild fibrosis within the muscularis propria layer (x100)

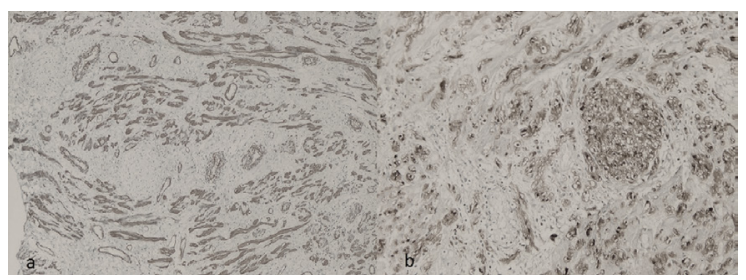

Fig. 6a,b. Neuronal hyperplasia lacking ganglion cells, perivascular lymphocytic infiltration and myocytolysis (H\&E; x100, x200)

after diagnosis revealed stable mural thickening detected in the initial diagnosis. The last examination ten years after the initial diagnosis revealed no change in the mural thickness. Written informed consent was received from the family for this case presentation.

\section{Discussion}

Primary chronic IPO involves the gastrointestinal system and can cause gastroparesis, dysphagia, diverticulosis, constipation, vomiting and hepatobiliary dilatation ${ }^{5}$. When primary IPO is caused by VM; hypokinetic and non-peristaltic oesophagus, gross duodenal enlargement, decreased small bowel contractions, megacystis, ptosis and ophthalmoplegia are expected, contrary to visceral neuropathy. ${ }^{2}$ Although the diagnosis was VM in the current case, there were not only decreased contractions but also mild mechanical obstruction due to obvious luminal stenosis due to mural thickening. In addition, megacystis, megaureter, urinary retention and recurrent urinary infections can be seen in cases of VM with urinary system involvement $t^{2}$. Diffuse mural thickening could be subdivided into focal (less than $5 \mathrm{~cm}$ ), segmental $(6$ to $40 \mathrm{~cm}$ ) or diffuse (longer than $40 \mathrm{~cm}$ ) involvement. ${ }^{6}$ Circumferential and symmetric thickening of the bowel walls, particularly with segmental and diffuse involvement, are expected from benign conditions such as inflammatory, infectious, oedematous and ischemic causes except intestinal lymphoma. ${ }^{6}$ 


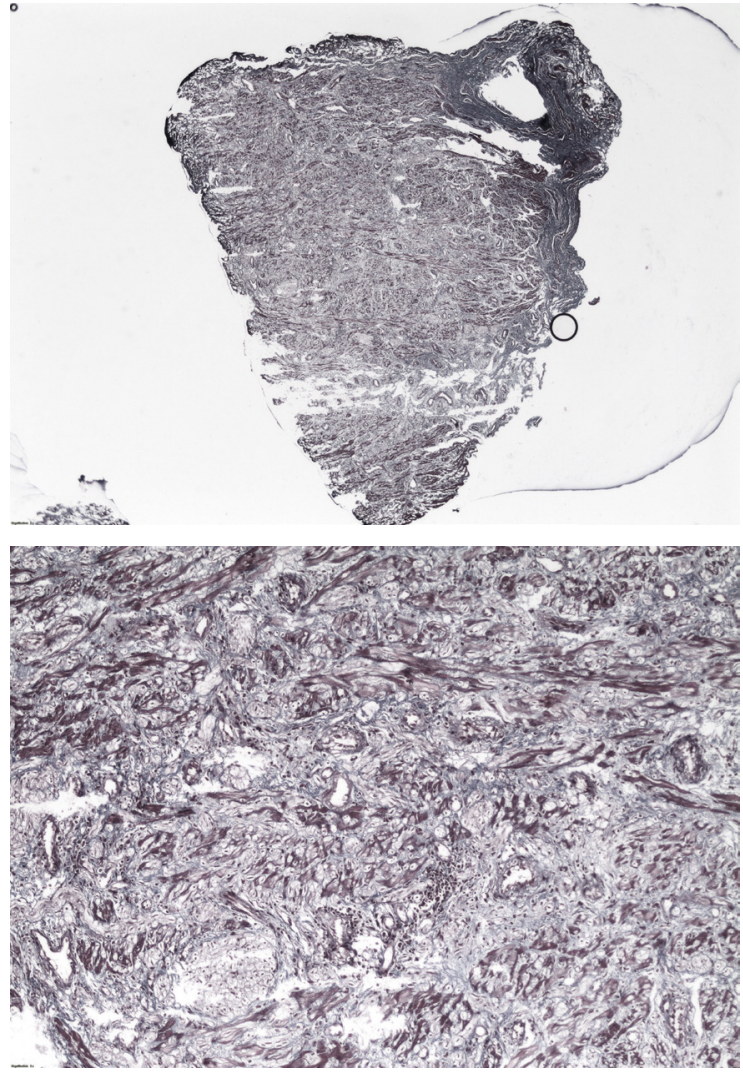

Fig. 7a. Smooth muscle actin immunohistochemistry highligts smooth muscle fibers (SMA IHC; x100) b. S100 immunoreactivity in hyperplastic nerve bundles (S100 IHC; $x 200)$

Double halo sign and target sign were described for stratified pattern of attenuation indicating inflammation or ischaemia after intravenous contrast administration but are nonspecific. ${ }^{7}$ Since VM is mostly reported as a cause of IPO, radiological findings have been reported as intestinal distension (mostly colonic) and presence of transitional zones without obstructive lesions on CT images. Given the findings in the presented case and a recent report $^{8}$, diffuse mural thickening with stratified pattern of attenuation in the absence of adjacent mesenteric inflammatory changes could be seen in cases with VM. In addition, moderate duodenal dilatation strengthened the presence of obstruction due to involvement of the jejunum with VM. Additionally, mesentery oedema and lymphangiectasia accompanied imaging findings and VM may be accepted as a cause of localized secondary lymphangiectasia. Indirect features on US such as dilatation of intestinal loops, regular and diffuse thickening of the walls, plical hypertrophy and severe mesenteric edema in some cases, ascites, may suggest primary intestinal lymphangiectasia in children ${ }^{9}$. The localized form of intestinal lymphangiectasia may occur not only as an associated imaging finding of $\mathrm{VM}$, but also in the differential diagnosis of VM. Both intestinal lymphangiectasia and VM are rare pathologies and should be confirmed with endoscopic findings and histopathological evaluation 10,11.

Imaging findings of Crohn Disease on US are noncompressible bowel wall, perienteric fluid and inflammation, creeping fat separating intestinal loops, fibrotic or inflammatory strictures, abscess and fistula ${ }^{12}$. Thickened intestinal wall is mostly seen in the terminal ileum in Crohn Disease ${ }^{13}$. Sectional evaluation may be necessary for extraintestinal additional findings. In our case the terminal ileum was intact, intestinal loops were compressible and mesenteric tissue was enlarged due to oedema. On DUS evaluation, increased superior mesenteric artery flow volume with decreased resistive index is reported to be correlated with Crohn Disease activity ${ }^{14}$. Contrary to this, we found a resistive index within normal range. Above all, perienteric fat planes in the presented case were not inflamed as expected from acute inflammatory bowel diseases.

Hirschsprung's disease (HD) is a strong differential diagnosis in patients with chronic constipation. A few reports included wall thickening and an echo-poor wall pattern of the sigmoid colon indicating HD $^{9}$, but HD is mostly reported with colonic dilatation. Blood flow velocities in celiac artery, superior mesenteric artery and inferior mesenteric artery were found to be increased in HD. Resistance to blood flow was found to be increased in the inferior mesenteric artery and decreased in the celiac artery and superior mesenteric artery ${ }^{10}$. Small bowel loops could be involved in HD in addition to colonic segments and a few cases were reported in the literature with skip segment HD. ${ }^{11}$ In addition small bowel involvement in HD is reported to be encountered less commonly. ${ }^{12}$ Intestinal lymphoma can be distinguished from inflammatory diseases and VM by obliteration of involved layers as well as the myenteric nerve plexus within the muscularis propria layer resulting in aneurysmal dilatation. Small bowel lymphoma is known to 
involve a single bowel loop. The mesenteric lymph nodes depicted on ultrasound were extirpated and histopathology report confirmed benign reactive changes and was considered to be caused by mesenteric congestion. Therefore, US has a significant value as an imaging modality by demonstrating the intestinal layers clearly with a higher geometrical resolution, narrowing differential diagnosis and excluding malignant conditions. The diagnosis of VM is established with the appearance of characteristic light microscopy findings after full-thickness intestinal biopsy in patients with suspicious clinical manifestations ${ }^{13}$. Typical findings on light microscopy are thinning, degeneration, and fibrous replacement in the smooth muscle cells of gastrointestinal tract. ${ }^{14}$ Unlike systemic sclerosis, which is accompanied by the involvement of external longitudinal layers of smooth muscle cells, VM is associated with vacuolar degeneration. ${ }^{15}$ The main purpose of VM therapy is to provide decompression with intravenous hydration and nutrition. ${ }^{16}$ Surgical resection and enterostomy are required if nasogastric and rectal tubes cannot provide ideal decompression. ${ }^{17}$

In cases of both diffuse or segmental mural thickening and intestinal dilatation without endoluminal obvious obstruction VM must be included in differential diagnosis as a cause of intestinal obstruction as well as palpable mass in a child. Inflammatory bowel diseases, intestinal lymphoma, and intestinal lymphangiectasia could be differential diagnoses in patients with multiple lymph nodes located in the mesenteric region in addition to intestinal wall thickening. Our case is the first case documenting US and MRI findings in a child who presented with a palpable abdominal mass and was diagnosed with VM.

\section{Acknowledgements}

The authors declare that they have all participated in the design, execution, and analysis of the paper, and that they have approved the final version. Additionally, there are no conflicts of interest in connection with this paper, and the material described is not published or under consideration for publication elsewhere. No financial support was received during the preparation of the paper.

\section{REFERENCES}

1. Georgescu EF, Vasile I, Ionescu R. Intestinal pseudo-obstruction: an uncommon condition with heterogeneous etiology and unpredictable outcome. World J Gastroenterol 2008; 14: 954-9.

2. Ghosh S, Eastwood MA. Primary chronic intestinal pseudo-obstruction--an update. Postgrad Med J 1994; 70: 65 .

3. Pal K, Moammar H, Mitra DK. Visceral myopathy causing chronic intestinal pseudoobstruction and intestinal failure in a child with Sanjad-Sakati syndrome. J Ped Surg 2010; 45: 430-434.

4. Burcharth J, Olsen C, Rosenberg J. Acute abdomen and perforated bowel with a rare pathology: Nonfamilial visceral myopathy. Case Rep Surg 2011; 2011: 645349.

5. De Giorgio R, Cogliandro RF, Barbara G, Corinaldesi R, Stanghellini V. Chronic intestinal pseudo-obstruction: clinical features, diagnosis, and therapy. Gastroenterol Clin North Am2011; 40: 787-807.

6. Macari M, Megibow AJ, Balthazar EJ. A pattern approach to the abnormal small bowel: 151 observations at MDCT and CT enterography. AJR Am J Roentgenol 2007; 188: 1344-1355.

7. Fernandes T, Oliveira MI, Castro R, Araujo B, Viamonte B, Cunha R. Bowel wall thickening at CT: simplifying the diagnosis. Insights Imaging 2014; 5: 195-208.

8. Reddy SB, Hamersk CM, Gavankar SS, et al. Sporadic visceral myopathy: Full thickness rectal biopsy to clinch the diagnosis. J Gastroenterol Hepatol Res 2012; 1: 161-164.

9. Maconi G, Molteni P, Manzionna G, Parente F, Porro GB. Ultrasonographic features of long-standing primary intestinal lympangiectasia. Eur J Ultrasound 1998; 7: 195-198.

10. Vignes S, Bellanger J. Primary intestinal lymphangiectasia (Waldmann's disease). Orphanet J Rare Dis. 2008;3:5.

11. Rohrmann Jr CA, Ricci MT, Krishnamurthy S, Schuffler MD. Radiologic and histologic differentiation of neuromuscular disorders of the gastrointestinal tract: visceral myopathies, visceral neuropathies, and progressive systemic sclerosis.AJR Am J Roentgenol., 1984; 143: 933-941.

12. Muradali D, Goldberg D. US of gastrointestinal tract disease. Radioghraphics 2015; 35: 50- 68.

13. Goulart RA, Barbalho SM, Gasparini RG, de Carvalho AC. Facing Terminal Ileitis: Going Beyond Crohn's Disease. Gastroenterology Res. 2016;9:1-9.

14. Yekeler E, Danalioglu A, Movasseghi B, Yilmaz S, Karaca C, Kaymakoglu S, Acunas B. Crohn disease activity evaluated by Doppler ultrasonography of the superior mesenteric artery and the affected small-bowel segments. J Ultrasound Med 2005; 24: 59-65.

15. Federmann G, Schneider A, Bauermeister G. Hypogangliosis of the sigmoid colon: possibilities of sonographic diagnosis. Ultraschall Med 1992; 13: 136-137.

16. Pratap A, Tiwari A, Shakya VC, et al. Doppler study of splanchnic hemodynamics in Hirschsprung's disease. Eur Surg Res 2007; 39: 148-152. 
17. Erten EE, Cavusoglu YH, Arda N, et al. A rare case of multiple skip segment Hirschsprung's disease in the ileum and colon. Pediatr Surg Int 2014; 30: 349-351.

18. Ziad F, Katchy KC, Al Ramadan S, Alexander S, Kumar S. Clinicopathological features in 102 cases of Hirschsprung disease. Ann Saudi Med 2006; 26: 200-204.

19. Moore SW, Schneider JW, Kaschula RDC. Non-familial visceral myopathy: Clinical and pathologic features of degenerative leiomyopathy. Pediatr Surg Int 2002; 18 : 6-12.

20. Martin JE, Benson M, Swash M, Salih V, Gray A. Myofibroblasts in hollow visceral myopathy: the origin of gastrointestinal fibrosis? Gut 1993; 34: 999-1001.
21. Smith JA, Hause SC, Madara JL. Hollow visceral myopathy: a light and electron microscopic study. Am J Surg Pathol 1982; 6: 269-275.

22. Pitt HA, Mann LL, Berquist WE. Chronic intestinal pseudo-obstruction. Management with total parenteral nutrition and a venting enterostomy. Arch Surg 1985; 120: 614-618.

23. Connor FL, Di Lorenzo C. Chronic intestinal pseudo-obstruction: assessment and management. Gastroenterology 2006; 130: 29-36. 